\title{
CARACTERIZAÇ̃̃o de CARVÃo ATIVAdo da PALHa de COQUEIRO E AVALIAÇÃO DO USO NA REMOÇÃO DE ÁCIDOS NAFTÊNICOS PRESENTES EM MISTURA MODELO DE QUEROSENE DE AVIAÇÃO
}

\author{
N. F. CAMPOS, J. H. L. dos SANTOS, C. M. B. M. BARBOSA, M.M.M.B. DUARTE \\ Universidade Federal de Pernambuco, Departamento de Engenharia Química e Engenharia Química \\ E-mail para contato: nataliaferreiracamp@ hotmail.com

\begin{abstract}
RESUMO - No petróleo são encontrados ácidos naftênicos que são causadores de corrosão em equipamentos. A adsorção destes ácidos é uma técnica promissora, pois possibilita a sua recuperação. Este trabalho teve como objetivo avaliar o uso do carvão ativado da palha de coqueiro (CA) para remoção de ácidos naftênicos presentes em mistura modelo de querosene de aviação. O CA foi ativado com fluxo de $100 \mathrm{~mL} \cdot \mathrm{min}^{-1}$ de $\mathrm{CO}_{2}$ à tempetura de $600^{\circ} \mathrm{C}$. Os carvões antes e após ativação foram classificados e caracterizados por medida da área superficial por adsorção de $\mathrm{N}_{2}$, difração de raios-X, termogravimétrica (TG) e definido o ponto de carga zero. Para o CA foi realizado planejamento fatorial $2^{2}$ para avaliar a influência da granulometria e da velocidade de agitação sobre a capacidade adsortiva $q$. Os carvões foram caracterizados como mesoporosos e apresentaram perfil do carbono grafite. No TG foi observado três perdas de massa. O CA obtive $q$ de $230 \mathrm{mg} \cdot \mathrm{g}^{-1}$ para o nível de $<0,09 \mathrm{~mm}$ e sem agitação, indicando potencial técnico para remoção de ácidos naftênicos.
\end{abstract}

\section{INTRODUÇÃO}

O petróleo é uma mistura complexa de hidrocarbonetos parafínicos, naftênicos, aromáticos e contaminantes tais como enxofre, nitrogênio, oxigênio, metais e ácidos naftênicos (Quelhas et al., 2012).

Os ácidos naftênicos também estão presentes nos derivados de petróleo. No querosene de aviação (QAV) que é um derivado do petróleo na faixa de ebulição de 150 e $300^{\circ} \mathrm{C}$, estes ácidos são responsavéis pela formação de compósitos e corrosão nos motores dos jatos (Farah, 2012).

Várias formas de resolver este problema já foram propostas, tais como: extração líquido-líquido (Shi et al., 2008), biodegradação (Quesnel et al., 2011), esterificação catalítica (Wang et al., 2014), porém, esses métodos apresentam custos elevados e problemas de operação.

A remoção dos ácidos através de processos de adsorção é uma técnica promissora, devido ao fato de a adsorção ser um processo não destrutivo, após remoção dos ácidos, eles podem ser purificados e comercializados (Gruber et al., 2012). 


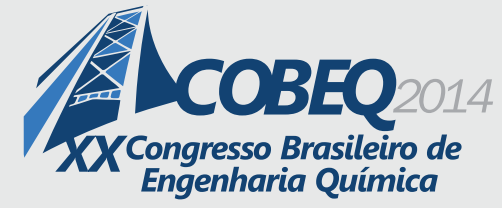

Dentre os adsorventes, o carvão ativado tem sido um dos mais empregados para remoção de compostos orgânicos provenientes do petróleo e seus derivados (Santos et al., 2007). É obtido industrialmente por pirólise oxidativa de madeira, carvão mineral, ossos e casca de coco. Suas propriedades de adsorção dependem do material precursor e também do método de ativação e carbonização (Gonzalez; Cid, 2005).

Neste intuito, pesquisadores estão estudando o desenvolvimento de novos carvões ativados a partir de resíduos agroindustriais, tais como cascas de nozes (Martínez et al., 2006), de mandioca (Sudaryanto et al., 2006), caroços de azeitonas (Martínez et al., 2006), de coco (Cazetta et al.; 2011), fibras de algodão (Sun et al., 2012) e resíduo de couro (Kong et al., 2013) para aplicações na remoção de compostos orgânicos.

O objetivo desse trabalho foi avaliar o uso do carvão da palha do coqueiro com e sem ativação para remoção de ácidos naftênicos presentes em mistura modelo de querosene de aviação.

\section{METODOLOGIA}

\subsection{Ativação do Carvão da Palha do Coqueiro}

O processo de ativação do carvão da palha do coqueiro ocorreu em duas etapas, inicialmente, o carvão foi submetido a uma rampa de aquecimento de $5 \mathrm{~K} \cdot \mathrm{min}^{-1}$, partindo da temperatura ambiente até $873 \mathrm{~K}$ em atmosfera inerte de nitrogênio a uma vazão de $100 \mathrm{~mL} \cdot \mathrm{min}^{-1}$. Após ter atingido a temperatura de $873 \mathrm{~K}$, o sistema permaneceu nesta condição por 60 minutos. Em seguida, o fluxo de nitrogênio foi trocado por $\mathrm{CO}_{2}$ na vazão de $100 \mathrm{~mL} \cdot \mathrm{min}^{-1}$ por tempo adicional de 60 minutos. Após o material atingir temperatura ambiente foram classificados numa série de peneiras de Tyler em três grupos de granulometrias $<0,09 \mathrm{~mm}, 0,09-0,15 \mathrm{~mm}$ e $0,15-0,21 \mathrm{~mm}$.

\subsection{Caracterização do Carvão da Palha do Coquerio com e sem Ativação}

Ponto de carga zero: $\mathrm{O}$ valor do $\mathrm{pH}$ no ponto de carga zero $\left(\mathrm{pH}_{\mathrm{pcz}}\right)$ dos carvões com e sem ativação foram estimados através da medida do $\mathrm{pH}$ da água antes e após contato com os sólidos. Nos ensaios foram utilizados $0,1 \mathrm{~g}$ do carvão para $25 \mathrm{~mL}$ de água com $\mathrm{pH}$ de 2 a 11 . $\mathrm{O} \mathrm{pH}$ das soluções foram ajustados em um pHmetro (Quimis, Q400AS) com $\mathrm{HCl}\left(0,1 \mathrm{~mol} . \mathrm{L}^{-1}\right)$ ou $\mathrm{NaOH}\left(0,1 \mathrm{~mol} . \mathrm{L}^{-1}\right)$. As soluções permaneceram sob agitação por 24 horas e depois foram filtradas para que o $\mathrm{pH}$ fosse medido. $\mathrm{O}$ gráfico $\Delta \mathrm{pH}\left(\mathrm{pH}_{\text {final }}-\mathrm{pH}_{\text {inicial }}\right)$ versus $\mathrm{pH}_{\text {inicial }}$ foi construído, e o valor do $\mathrm{pH}_{\mathrm{pcz}}$ foi estimado através deste gráfico.

Caracterização textural: A caracterização dos carvões foi realizada por adsorção/dessorção de nitrogênio através do método de BET para determinação de área superficial específica e o método BJH para obtenção do volume dos poros do carvão. A área superficial do material foi determinada através da adsorção de $\mathrm{N}_{2}$ a $77 \pm 5 \mathrm{~K}$ em um equipamento Micromeritics (modelo ASAP 2420). Inicialmente, 0,2 $\mathrm{g}$ de amostra foi pré-tratada a $333 \mathrm{~K}$ sob vácuo (DEGASS) por 3 horas.

Difraçã de raio-X: Na análise de difração de raios-X (DRX), os carvões foram caracterizadas 


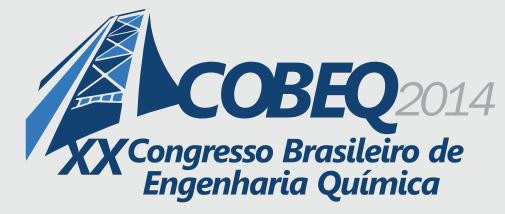

em um Difratômetro de Raios-X BRUKER (modelo D8 ADVANCE) utilizando-se uma fonte de radiação de $\mathrm{Cu}-\mathrm{K} \alpha$ com voltagem de $30 \mathrm{kV}$, corrente de $30 \mathrm{~mA}$. Os dados foram coletados na faixa de $2 \theta$ de $5^{\circ}$ a $80^{\circ}$ com passo de $0,05^{\circ}$ e passo no tempo de $2,0 \mathrm{~s}$.

Análise termogravimétrica: A curva termogravimétrica foi obtida em uma termobalança NETZSCH (modelo STA 449 F3 Jupiter), em atmosfera de $100 \mathrm{~mL} \cdot \mathrm{min}^{-1}$ de nitrogênio. Nesta análise, foram utilizados cadinhos de alumina, razão de aquecimento de $20 \mathrm{~K} . \mathrm{min}^{-1}$, massa da amostra de 6,0 $\pm 0,5 \mathrm{mg}$ em intervalo de temperatura de 313 a $873 \mathrm{~K}$.

Solução do ácido naftênico: A mistura modelo foi preparada pesando-se $2,25 \mathrm{~g}$ do ácido carboxílico n-dodecanóico que foi dissolvido com o solvente n-dodecano, a mistura foi transferida para um balão de $100 \mathrm{~mL}$ e o volume foi aferido, obtendo-se uma concentração de $3 \%(\mathrm{~m} / \mathrm{m})$. O ácido carboxílico n-dodecanóico foi selecionado como composto modelo para representar os ácidos naftênicos. Este ácido foi usado nos testes de adsorção devido às suas propriedades físicas e similaridade com os compostos ácidos frequentemente encontrados no QAV (NASCIMENTO, 2011).

\subsection{Definição das Condições de Trabalho}

Um planejamento fatorial foi realizado para avaliar as influências das variáveis, granulometria (mm) e velocidade de agitação (rpm), sobre o processo adsortivo (Tabela 1). Os ensaios foram realizados em ordem aleatória. O planejamento fatorial utilizado foi do tipo $2^{2}$ com ponto central em triplicata, de forma a garantir a reprodutibilidade dos dados experimentais. A resposta utilizada para determinar a eficiência do processo foi a capacidade adsortiva, $q\left(\mathrm{~g} \cdot \mathrm{g}^{-1}\right)$.

Tabela 1 - Níveis dos fatores utilizados para o planejamento fatorial $2^{2}$ com ponto central..

\begin{tabular}{|c|c|c|c|}
\hline VARIÁVEL & \multicolumn{3}{|c|}{ NÍVEIS } \\
\hline & -1 & 0 & 1 \\
\hline Granulometria - G (mm) & $<0,09$ & $0,09-0,15$ & $0,15-0,21$ \\
\hline Velocidade de agitação - V (rpm) & 0 & 150 & 300 \\
\hline
\end{tabular}

Para realização dos ensaios foram utilizados erlenmeyers contendo $0,05 \mathrm{~g}$ do carvão ativado (CA) permaneceram em contato com $5 \mathrm{~mL}$ da solução modelo de querosene de aviação durante 360 minutos à temperatura ambiente do laboratório $\left(25^{\circ} \mathrm{C} \pm 2^{\circ} \mathrm{C}\right)$, com auxílio da mesa agitadora. Foram realizados ensaios independentes para os três carvões, bem como ensaios em branco.

Os cálculos dos efeitos dos fatores e as interações entre eles com os seus respectivos erros padrão foram realizados segundo Barros Neto; Scarminio; Bruns (2007), com auxilio do programa Statistica 6.0.

\section{RESULTADOS E DISCUSSÃO}

\subsection{Caracterização do Carvão da Palha do Coquerio com e sem Ativação}


Ponto de carga zero: A Figura 1 apresenta o gráfico $\Delta \mathrm{pH}\left(\mathrm{pH}_{\text {final }}-\mathrm{pH}_{\text {inicial }}\right)$ versus $\mathrm{pH}_{\text {inicial }}$ para os carvões da palha do coqueiro com e sem ativação.

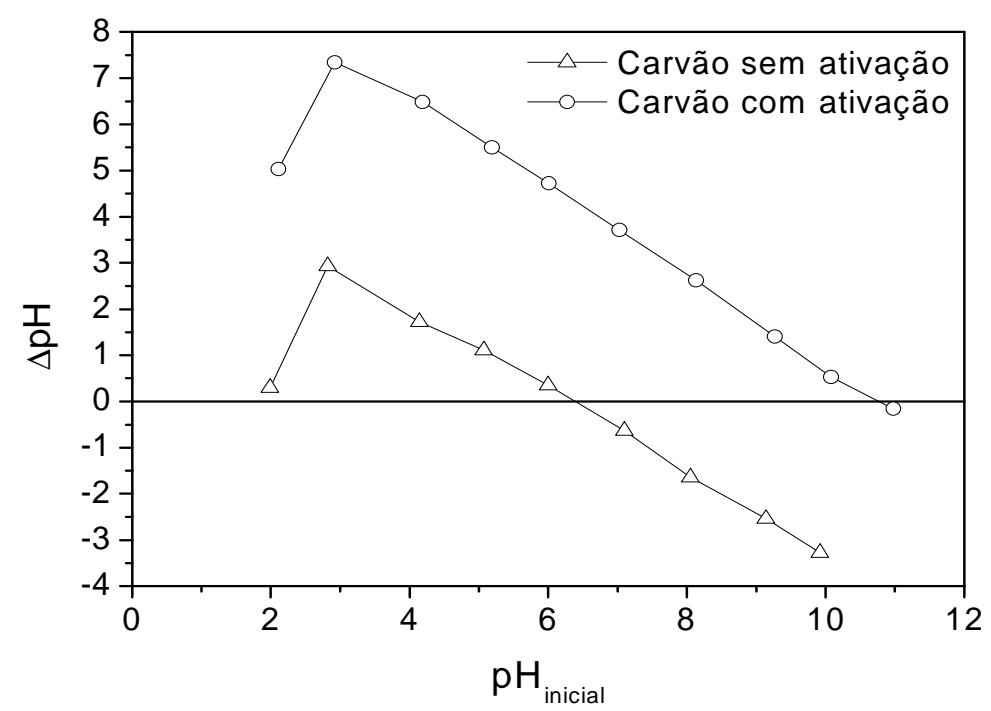

Figura 1 - Ponto de carga zero dos carvões da palha do coqueiro com e sem ativação.

Segundo Nascimento et al. (2014), abaixo do $\mathrm{pH}_{\mathrm{pcz}}$, o material apresenta cargas positivas na superfície favorecendo a adsorção de ânions, e consequentemente, acima do $\mathrm{pH}_{\mathrm{pcz}}$, o material apresenta cargas negativas na superfície promovendo a adsorção de cátions.

De acordo com a Figura 1, os carvões com e sem ativação apresentaram $\mathrm{pH}_{\mathrm{pcz}}$ igual a 6,4 e 10,7, respectivamente. Este resultado indica que o carvão após ativação apresenta carga positiva em suas superfícies em uma ampla faixa de $\mathrm{pH}$, corroborando com Rodrigues et al. (2011) que obtiveram para carvão ativado preparado a partir do caroço do abacate ativado com $\mathrm{CO}_{2} \mathrm{pH}_{\mathrm{pcz}}$ igual a 9,7.

Caracterização textural: Os resultados obtidos para a caracterização textural por adsorção/dessorção de $\mathrm{N}_{2}$ estão apresentados na Tabela 2.

Tabela 2 - Resultados obtidos por adsorção/dessorção de $\mathrm{N}_{2}$ para os carvões.

\begin{tabular}{|c|c|c|c|}
\hline CARVÃO & $\begin{array}{c}\text { ÁREA SUPERFICIAL } \\
\left(\mathrm{m}^{2} \cdot \mathrm{g}^{-1}\right)\end{array}$ & $\begin{array}{c}\text { VOLUME DO PORO } \\
\left(\mathrm{cm}^{3} \cdot \mathrm{g}^{-1}\right)\end{array}$ & $\begin{array}{c}\text { DIÂMETRO DO PORO } \\
(\AA)\end{array}$ \\
\hline Carvão sem ativação & 155,7 & 0,03 & 55,2 \\
\hline Carvão com ativação & 258,5 & 0,15 & 23,0 \\
\hline
\end{tabular}

De acordo com a União Internacional de Química Pura e Aplicada (IUPAC), materiais com tamanho de poro no intervalo de 20-500 A são classificados como mesoporosos.

Segundo Matos et al. (2011), materiais carbonáceos após ativação com $\mathrm{CO}_{2}$ apresentam um 


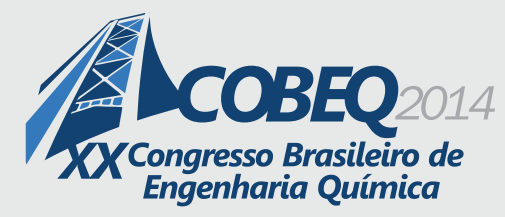

aumento na área superficial e no volume do poro, e uma diminuição do diâmetro do poro, comportamento observado neste trabalho conforme Tabela 2.

Resultados semelhantes foram obtidos por Rodrigues et al. (2011) que obtiveram área superficial igual a $206 \mathrm{~m}^{2} \cdot \mathrm{g}^{-1} \mathrm{e}$ volume de poro igual a $0,1 \mathrm{~cm}^{3} \cdot \mathrm{g}^{-1}$ para o carvão do caroço de abacate ativado com $\mathrm{CO}_{2}$.

Difraçã de raio-X: Os carvões apresentaram o mesmo padrão de DRX, uma vez que foram preparados a partir do mesmo resíduo agroindustrial. O difratograma obtido está apresentado na Figura 2.

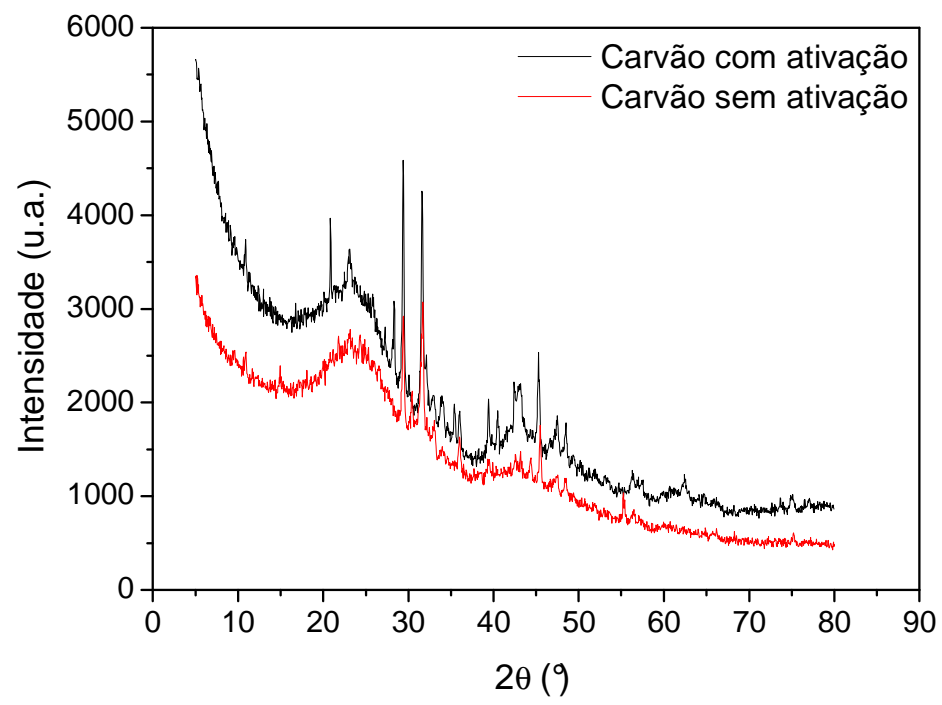

Figura 2 - Difratograma de raio-X dos carvões da palha do coqueiro com e sem ativação.

$\mathrm{Na}$ Figura 2, observam-se picos finos no difratograma, sobrepostos aos dois halos (aproximadamente $24,3^{\circ}$ e $43,3^{\circ}$ ), evidenciam a presença de partículas cristalinas no material, que se constituem em resíduos produzidos no processo de carbonização. Os halos naquelas posições são característicos do perfil de difração do carbono grafite e são atribuídos às reflexões dos planos (002) e (101). Este último representa uma sobreposição das reflexões dos planos 100 e 101. Resultados semelhantes foram obtidos por Macedo (2005).

Ainda na Figura 2 verifica-se que o carvão com ativação apresentou maior intensidade sendo indicativo de uma maior organização do material.

Análise termogravimétrica: Os carvões com e sem ativação apresentaram três perdas de massa, duas bem definidas e uma mais discreta. A primeira perda de massa, abaixo de $200{ }^{\circ} \mathrm{C}$ deve-se a perda de água higroscópica. A segunda perda de massa é referente à degradação dos grupos com oxigênio da superfície do carvão. Em seguida, verifica-se a terceira perda de massa, devido aos grupos carboxílicos que são menos estáveis e se decompoem a $400^{\circ} \mathrm{C}$. 
Este resultado corrobora com os obtidos por Guimarães (2006), que na análise termogravimétrica observou três perdas de massa para o carvão do endocarpo de coco. Segundo o mesmo autor, o TG mostra que os grupos com oxigênio da superfície de carvões são termicamente estáveis até $200^{\circ} \mathrm{C}$, independente do processo de preparação.

\subsection{Definição das Condições de Trabalho}

A partir dos dados experimentais e com auxilio do programa Statistica 6.0 foram calculados os efeitos principais e de interação (Tabela 3) bem como construídas o gráfico de pareto e as superfícies de respostas para o carvão da palha do coqueiro com ativação. Os efeitos que são considerados estatisticamente significativos a um nível de $95 \%$ de confiança nos níveis estudados, são aqueles além da linha do gráfico de Pareto $(\mathrm{p}=0,05)$.

Tabela 3 - Efeitos principais e de interação calculados para o planejamento fatorial $2^{2}$. Efeito estatisticamente significativo em nível de $95 \%$ de confiança é mostrado em negrito.

\begin{tabular}{|c|c|}
\hline EFEITOS & NÍVEIS \\
\hline Média & $0,032 \pm 0,003$ \\
\hline Efeitos principais: & \\
\hline Granulometria - G (mm) & $\mathbf{- 0 , 0 2 9 \pm 0 , 0 0 6}$ \\
\hline Velocidade de agitação - V (rpm) & $-0,012 \pm 0,006$ \\
\hline Interação de dois fatores: & \\
\hline G vs. VA & $\mathbf{0 , 0 4 9} \pm \mathbf{0 , 0 0 6}$ \\
\hline
\end{tabular}

Para o CA da palha do coqueiro, o efeito Granulometria e a interação entre os efeitos Granulometria e Velocidade de Agitação foram estatisticamente significativos. Podendo ser melhor visualizado através da carta de Pareto na Figura 4A. Como à interação de dois fatores estatisticamente significativos, esta interação deve ser analisado através da superfície de resposta que está apresentada na Figura 4B. 

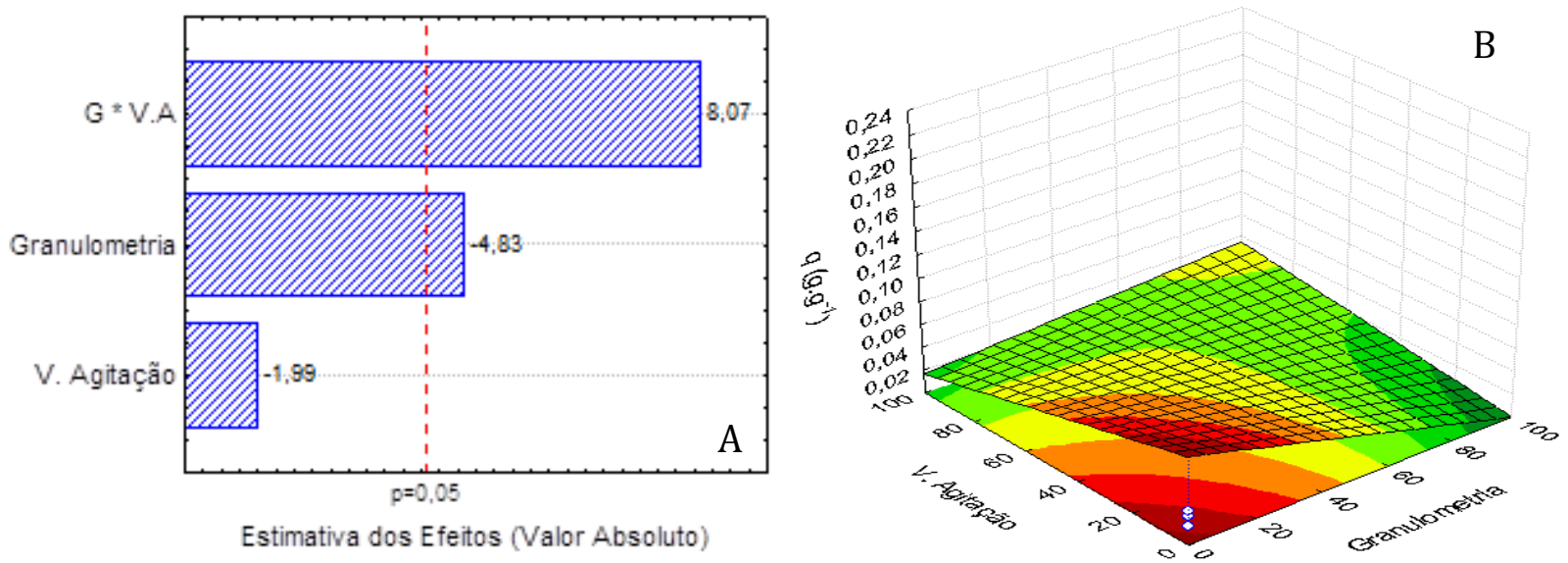

Na Figura 4B, pode-se observar que para o CA da palha do coqueiro, a maior capacidade adsortiva foi obtida para o nível de $<0,09 \mathrm{~mm}$ e sem agitação.

\section{CONCLUSÃO}

Pode-se que concluir que a ativação o carvão da palha do coqueiro com $\mathrm{CO}_{2}$ promoveu uma ampla faixa de carga positiva na superfície, maior área superficial e volume do poro, maior organização do material, sendo refletido na capacidade adsortiva obtida igual a $230 \mathrm{mg} \cdot \mathrm{g}^{-1}$.

\section{AGRADECIMENTOS}

Ao programa PRH-28/ANP; À PETROBRAS; Ao CETENE e Elephant Indústria Química Ltda.

\section{REFERENCIAS}

BARROS NETO, B., SCARMINIO, I. S., BRUNS, R. E. Como Fazer Experimentos: Pesquisa e desenvolvimento na ciência e na industria. Campinas: Editora Unicamp, 2007.

CAZETTA, A.L.; VARGAS, A.M.M.; NOGAMI, E.M.; KUNITA, M.H.; GUILHERME, M.R.; MARTINS, A.C.; SILVA, T.L.; MORAES, J.C.G.; ALMEIDA, V.C. NaOH-activated carbon of high surface area produced from coconut shell: Kinetics and equilibrium studies from the methylene blue adsorption. Chem. Eng. J., v. 174, p. 117-125, 2011.

FARAH, M.A. Petróleo e seus derivados. Rio de Janeiro: Editora LTC, 2012.

GONZALEZ, M. F. E.; CID, A. A. P. Adsorbentes de origen natural para laeliminación de colorantes textiles. In: REINOSO, F. R. Descontaminación Ambiental Mediante Adsorbentes. Espanha: Editora CYTED, 2005.

GRUBER, L. D. A.; DAMASCENO, F. C.; CARAMÃO, E. B.; JACQUES, R. A.; GELLER, A. M.; DE CAMPOS, M. C. V. Ácidos naftênicos no petróleo. Quim. Nova, v. 35, p. 1423-1433, 2012. 


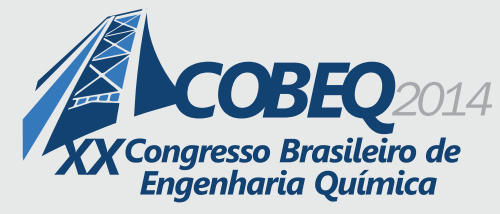

GUIMARÃES, I. S. Oxidação de carvões ativados de endocarpo de coco de Baía com soluções de $\mathrm{HNO}_{3}$ e uma investigação sobre o método de Boehm. Dissertação de Mestrado. João Pessoa, PB: Universidade Federal da Paraíba, 2006. 81p.

KONG, J.; YUE, Q.; HUANG, L.; GAO, Y.; SUN, Y.; GAO, B.; LI, Q.; WANG, Y. Preparation, characterization and evaluation of adsorptive properties of leather waste based activated carbon via physical and chemical activation. Chem. Eng. J., v. 221, p. 62-71, 2013.

MACEDO, J. S. Aproveitamento dos resíduos do beneficiamento de fibras de coco na obtenção de um eco-material: carbono ativado mesoporoso. Dissertação de Mestrado. São Cristóvão, SE: Universidade Federal de Sergipe, 2005. 106f.

MARTÍNEZ, M.L.; TORRES, M.M; GUZMÁN, C.A.; MAESTRI, D.M. Preparation and characteristics of activated carbon from olive stones and walnut shells. Ind. Crop. Prod., v. 23, p. 23$28,2006$.

NASCIMENTO, G. E. Remoção de ácidos naftênicos em mistura modelo de querosene de aviação (ácido ndodecanóico em n-dodecano) por adsorção, utilizando novos materiais. Dissertação de Mestrado. Recife, PE: Universidade Federal de Pernambuco, 2011. 106p.

NASCIMENTO, G. E.; DUARTE, M. M. M. B.; CAMPOS, N. F.; ROCHA, O. R. S.; SILVA, V. L. Adsorption of azo dyes using peanut hull and orange peel: a comparative study. Environ. Technol., v.35, p. 1436-1453, 2014.

QUELHAS, A.D.; BRASIL, N.I.; ARAÚJO, M.A.S.; SOUSA, E.C.M. Processamento de Petróleo e Gás: petróleo e seus derivados, processamento primário, processos de refino, petroquímica, meio ambiente. Rio de Janeiro: Editora LTC, 2012.

QUESNEL, D. M.; BHASKAR, I. M.; GIEG, L. M.; CHUA, G. Naphthenic acid biodegradation by the unicellular alga Dunaliella tertiolecta. Chemosphere, v.84, p. 504-511, 2011.

RODRIGUES, L. A. SILVA, M. L. AlvareZ-MENDES, M. O. COUTINHO, A. R. THIM, G. P. Phenol removal from aqueous solution by activated carbon produced from avocado kernel seeds. Chem. Eng. J., v. 174, p. 49-57, 2011.

SANTOS, E.G.; ALSINA, O.L.S.; SILVA, F.L.H. Desempenho de biomassas na adsorção de hidrocarbonetos leves em efluentes aquosos. Quim. Nova, v. 30, p. 327-331, 2007.

SHI, L.J.; SHEN, B.X.; WANG, G.Q. Removal of naphthenic acids from beijing crude oil by forming ionic liquids. Energ. Fuel, v.22, p. 4177-4181, 2008.

SUDARYANTO, Y.; HARTONO, S.B.; IRAWATY, W.; HINDARSO, H.; ISMADJI, S. High surface area activated carbon prepared from cassava peel by chemical activation. Bioresource Technol., v. 97, p. 734-739, 2006.

SUN, Y.; YUE, Q.; GAO, B.; LI, Q.; HUANG, L.; YAO, F.; XU, X. Preparation of activated carbon derived from cotton linter fibers by fused $\mathrm{NaOH}$ activation and its application for oxytetracycline (OTC) adsorption. J. Colloid Interf. Sci., v. 368, p. 521-527, 2012.

WANG, Y.; LI, J.; SUN, X.; DUAN, H.; SONG, C.; ZHANG M.; LIU Y. Removal of naphthenic acids from crude oils by fixed-bed catalytic esterification. Fuel, v. 116, p. 723-728, 2014. 